Original Research Paper

\title{
A New Extension of the Burr Type XII Distribution
}

\author{
Mohamed K.A. Refaie \\ Al-Ajami Higher Institute for Administrative Sciences, Alexandria, Egypt
}

Article history

Received: 10-11-2018

Revised: 26-11-2018

Accepted: 12-12-2018

Email: refaie_top@yahoo.com

\begin{abstract}
In this study, a new Burr XII distribution is defined and studied. Various structural mathematical properties of the proposed model are investigated. The maximum likelihood method is used to estimate the model parameters. We assess the performance of the MLEs of the new distribution with respect to sample size $n$. The assessment was based on a simulation study. The new distribution is applied for modeling two real data sets to prove empirically its flexibility. The new Burr XII model can be viewed as a suitable model for fitting the right skewed and unimodal data. The new model provides adequate fits as compared to other Burr XII models by means of two applications.
\end{abstract}

Keywords: Burr XII Distribution, Burr-Hatke Distribution, Simulation, Moments, Maximum Likelihood Method

\section{Introduction}

Burr (1942) introduced another new system of frequency curves, analogously to the Pearson systemof densities, that includes twelve types of Cumulative Distribution Function (CDFs) which yield avariety of density shapes, this system is obtained by considering CDFs satisfying a differentialequation which has a solution, given by:

$$
G(x)=\left\{1+\exp \left[-\int \Upsilon(x) d x\right]\right\}^{-1},
$$

where, $\Upsilon(x)$ is chosen such that $G(x)$ is a CDF on the real line and has twelve choices which madeby Burr, resulted in twelve models which might be useful for modeling data, the principal aim inchoosing one of these forms of distributions is to facilitate the mathematical analysis to which itis subjected, while attaining a reasonable approximation. A special attention has been devoted toone of these forms denoted by type XII whose CDF, $G(x)$; is given as:

$$
G_{\alpha, \beta}(x)=1-\left(x^{\alpha}+1\right)-\beta,
$$

both $\alpha$ and $\beta$ are shape parameters, location and scale parameters can easily be introduced tomake (1) a fourparameter distribution. The corresponding Probability Density Function (PDF) isgiven by:

$$
g_{\alpha, \beta}(x)=\alpha \beta x^{\alpha-1}\left(x^{\alpha}+1\right)^{-\beta-1} \text {, }
$$

The Burr XII Distribution (BXIID) originally proposed by Burr (1942), it has many applicationsin different areas. Coming early, Tadikamalla (1980) studied the BXIID and its related models. Some important extensions of the BXIID can be cited by Shao (2004), Zimmer et al. (1998), Soliman (2005), Wu et al. (2007), Silva et al. (2008), Silva et al. (2010a; 2010b), Cordeiro et al. (2018), Afify et al. (2018), Altun et al. (2018a; 2018b) and Yousof et al. (2018a; 2018b). (for more details about the BXIID see Burr (1942), (1968) and (1973), Burr and Cislak (1968), Hatke (1949) and Rodriguez (1977)). In this study, we propose a new BXII distributions, called the Burr-Hatke Exponentiated BXII Distribution (BHEBXII) by means of Burr-Hatke differential equation. In statistical literature, the so-called Burr-Hatke differential equation can be given asfollows:

$\frac{d}{d t} F=\left.g(t, F) F(1-F)\right|_{\left(F_{0}=F\left(t_{0}\right), t_{0} \in \Re\right)}$,

where, $F=F(t)$ is the Cumulative Distribution Function (CDF) of a continuous random variable $T$ and $g(t, F)$ is an arbitrary positive function for any $t_{0} \in \mathfrak{R}$. Equation (1) is considered bymany authors as a system of $\mathrm{CDF}(\mathrm{s})$ generator or simply a system of frequency curves. Using (1), Maniu and Voda (2008) introduced and studied the Burr-Hatke Distribution (BHD) with CDF and Probability Density Function (PDF) given by:

$$
F(t ; \theta)=1-\left.(t+1)^{-1} \exp (-t \theta)\right|_{(t>0, \theta>0)},
$$


and:

$$
f(t ; \theta)=\left.(t+1)^{-2} \exp (-t \theta)[\theta(t+1)+1]\right|_{(t>0, \theta>0)}
$$

respectively. Following Yousof et al. (2018) and replacing t by $\left\{-\log \left[\bar{G}_{b, \alpha, \beta}(x)\right]\right\}$, where $\bar{G}_{b, \alpha, \beta}(x)=\left[1-G_{b, \alpha, \beta}(x)\right]$ and:

$$
G_{b, \alpha, \beta}(x)=\left[1-\left(x^{\alpha}+1\right)^{-\beta}\right]^{b}
$$

is the CDF of the EBXIID. The CDF of the Burr-Hatke EBXII distribution (BHEBXIID) isdefined by:

$$
\begin{aligned}
& F_{\theta, b, \alpha, \beta}(x) \\
& =1-\left\{1-\left[1-\left(x^{\alpha}+1\right)^{-\beta}\right]^{b}\right\}^{\theta}\left(1-\log \left\{1-\left[1-\left(x^{\alpha}+1\right)^{-\beta}\right]\right\}^{b}\right)^{-1} .
\end{aligned}
$$

The PDF corresponding to (2) is given by:

$$
\begin{aligned}
& f_{\theta, b, \alpha, \beta}(x)=b \alpha \beta x^{\alpha-1}\left[1-\left(x^{\alpha}+1\right)^{-\beta}\right]^{b-1} \\
& \times\left(1-\log \left\{1-\left[1-\left(x^{\alpha}+1\right)^{-\beta}\right]^{b}\right\}\right)^{-2} \\
& \times\left(x^{\alpha}+1\right)^{-\beta-1}\left\{1-\left[1-\left(x^{\alpha}+1\right)^{-\beta}\right]^{b}\right\}^{\theta-1} \\
& \times\left\{\theta\left[1-\log \left\{1-\left[1-\left(x^{\alpha}+1\right)^{-\beta}\right]^{b}\right\}\right]+1\right\} .
\end{aligned}
$$

The Reliability Function (RF) and Hazard Rate Function (HRF) of new BH-G family are given by:

$$
\begin{aligned}
& R_{\theta, b, \alpha, \beta}(x) \\
& =\left\{1-\left[1-\left(x^{\alpha}+1\right)^{-\beta}\right]^{b}\right\}^{\theta}\left(1-\log \left\{1-\left[1-\left(x^{\alpha}+1\right)^{-\beta}\right]^{b}\right\}\right)^{-1},
\end{aligned}
$$

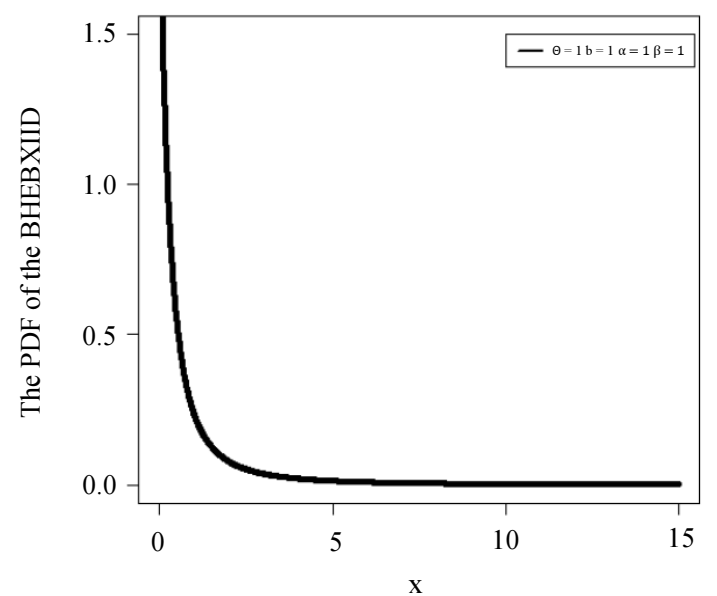

and:

$$
\begin{aligned}
& h_{\theta, b, \alpha, \beta}(x)=b \alpha \beta x^{\alpha-1}\left(x^{\alpha}+1\right)^{-\beta-1}\left[1-\left(x^{\alpha}+1\right)^{-\beta}\right]^{b-1} \\
& \times \frac{\left[\theta\left(1-\log \left\{1-\left[1-\left(x^{\alpha}+1\right)^{-\beta}\right]^{b}\right\}\right)+1\right]}{\left\{1-\left[1-\left(x^{\alpha}+1\right)^{-\beta}\right]^{b}\right\}\left(1-\log \left\{1-\left[1-\left(x^{\alpha}+1\right)^{-\beta}\right]^{b}\right\}\right)} .
\end{aligned}
$$

Figure 1 displays some plots of the new density for some parameter values. Plots of the HRF of thenew model for selected parameter values are given in Fig. 2, where the HRF can be decreasing, increasing and unimodal.

We are motivated to introduce the BHEBXIID because it exhibits the decreasing, increasing and unimodal HRF as illustrated in Fig. 2. It is shown in Subsection 2.2 that the BHEBXIID can be viewed as a linear mixture of the BXII densities as illustrated in Equations (6) and (7). It can be viewed as a suitable model for fitting the unimodal and right skewed data as illustrated in Section 4. The BHEBXIID provide adequate fits as compared to other BXIIDs by means of two applications with small values for AIC, BIC, CAIC and HQIC. The proposed BHEBXIID is much better than the BXIID, Marshall. Olkin Burr XII (MOBXIID), TL Burr XII, Kumaraswamy Burr XII (KwBXIID), beta Burr XII (BBXIID), Beta Exponentiated Burr XII (BEBXIID), Five parameter beta Burr XII (FBBXIID), Five parameter Kumaraswamy Burr XII(FKwBXIID) and Zografos-Balakrishnan Burr XII (ZBBXIID) in modeling the breaking stress and the taxes revenue data.

The rest of the paper is outlined as follows. In section 2 , we derive some statistical properties for the new model. Maximum likelihood estimation of the model parameters is addressed in section 3. Section 5 provides the simulation results. Two applications to real data sets to illustrate the importance of the new model are provided in section 5 . Finally, we offer some concluding remarks in section 6.

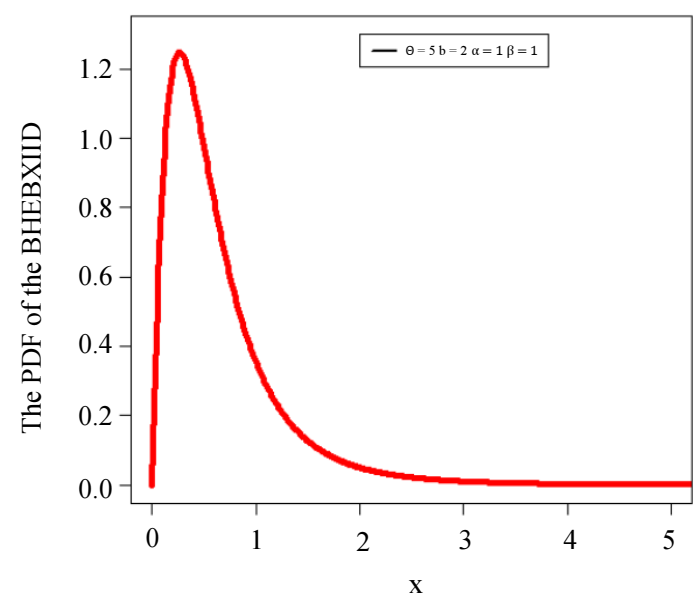



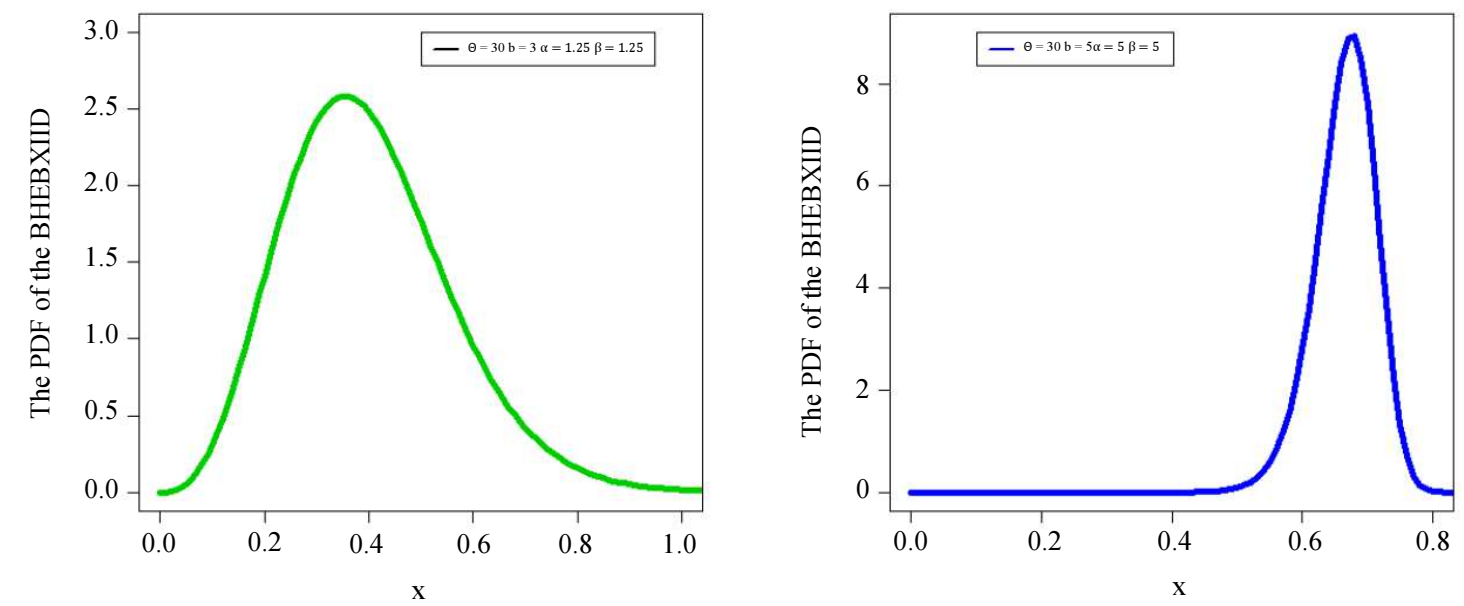

Fig. 1: Plots of the BHEBXII PDF
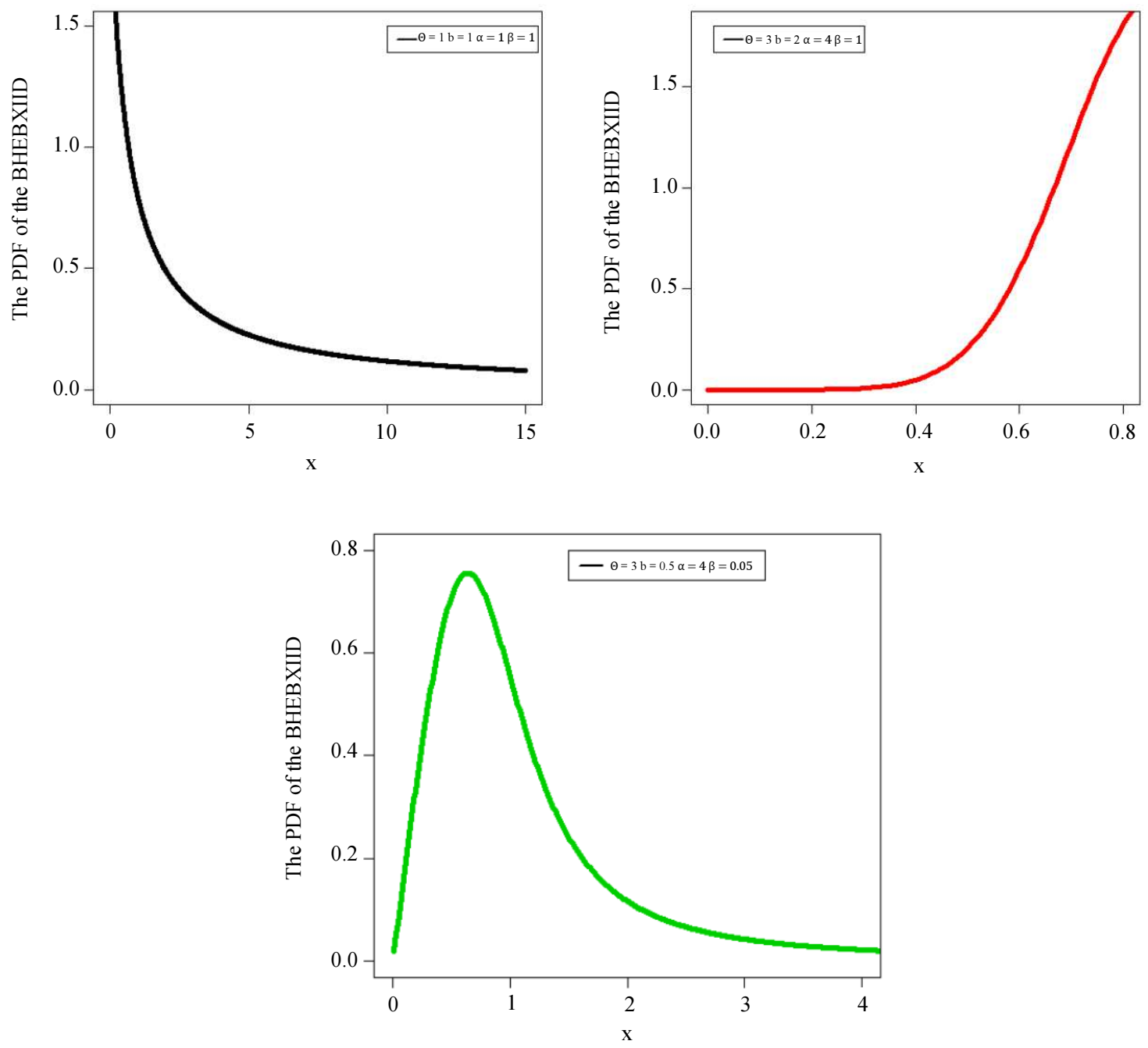

Fig. 2: Plots of the BHEBXII HRF 


\section{Properties}

\section{Asymptotics}

Let $a=\inf \left\{x \mid F_{\theta, b, \alpha, \beta}(x)>0\right\}$ the asymptotics of CDF, PDF and HRF as $x \rightarrow a$ are given by:

$$
\begin{aligned}
& \left.F_{\theta, b, \alpha, \beta}(x) \sim\left[1-\left(x^{\alpha}+1\right)^{-\beta}\right]^{b}\right|_{(x \rightarrow a)}, \\
& \left.f_{\theta, b, \alpha, \beta}(x) \sim b \alpha \beta x^{\alpha-1}\left(x^{\alpha}+1\right)^{-\beta-1}\left[1-\left(x^{\alpha}+1\right)^{-\beta}\right]^{b-1}\right|_{(x \rightarrow a)}
\end{aligned}
$$

and:

$$
\left.h_{\theta, b, \alpha, \beta}(x) \sim b \alpha \beta x^{\alpha-1}\left(x^{\alpha}+1\right)^{-\beta-1}\left[1-\left(x^{\alpha}+1\right)^{-\beta}\right]^{b-1}\right|_{(x \rightarrow a)} .
$$

The asymptotics of CDF, PDF and HRF as $x \rightarrow \infty$ are given by:

$$
\begin{aligned}
& 1-F_{\theta, b, \alpha, \beta}(x) \sim-\frac{\left\{1-\left[1-\left(x^{\alpha}+1\right)^{-\beta}\right]^{b}\right\}^{\theta}}{\log \left\{1-\left(1-\left(x^{\alpha}+1\right)^{-\beta}\right) b\right\}_{(x \rightarrow \infty)}} \\
& f_{\theta, b, \alpha, \beta}(x) \sim b \alpha \beta x^{\alpha-1}\left(x^{\alpha}+1\right)^{-\beta-1} \\
& {\left[1-\left(x^{\alpha}+1\right)^{-\beta}\right]^{b-1}\left\{1-\left[1-\left(x^{\alpha}+1\right)^{-\beta}\right]^{b}\right\}^{\theta-1}} \\
& \times\left(-\log \left\{1-\left[1-\left(x^{\alpha}+1\right)^{-\beta}\right]^{b}\right\}\right)^{-2} \\
& \left.\left(1+\theta \log \left\{1-\left[1-\left(x^{\alpha}+1\right)\right]^{-\beta}\right\}^{b}\right)^{1}\right|_{(x \rightarrow \infty)}
\end{aligned}
$$

and:

$$
\begin{aligned}
& h_{\theta, b, \alpha, \beta}(x) \sim b \alpha \beta x^{\alpha-1}\left(x^{\alpha}+1\right)^{-\beta-1} \\
& {\left[1-\left(x^{\alpha}+1\right)^{-\beta}\right]^{b-1}\left\{1-\left[1-\left(x^{\alpha}+1\right)^{-\beta}\right]^{b}\right\}^{-1}} \\
& \times\left(-\log \left\{1-\left[1-\left(x^{\alpha}+1\right)^{-\beta}\right]^{b}\right\}\right)^{-1} \\
& \left.\left(1+\theta \log \left\{1-\left[1-\left(x^{\alpha}+1\right)^{-\beta}\right]\right\}\right)\right|_{(x \rightarrow \infty)} .
\end{aligned}
$$

The effect of the parameters on tails of distribution can be evaluated by means of above equations.

\section{Useful Expansions}

In this section, mixture representations for Equations (2) and (3) are obtained. Consider the following expansions:

$$
\left.(1-z)^{t}\right|_{(|z|<\mid)}=\sum_{k=0}^{\infty}(-1)^{k}\left(\begin{array}{l}
t \\
k
\end{array}\right) z^{k}
$$

and:

$$
\left.\log (1-z)\right|_{(z \mid<1)}=-\sum_{k=0}^{\infty} \frac{z^{k-1}}{(k+1)} .
$$

Applying (4) for:

$$
\left\{1-\left[1-\left(x^{\alpha}+1\right)^{-\beta}\right]^{b}\right\}^{\theta}
$$

in Equation (2) we get:

$$
\left\{1-\left[1-\left(x^{\alpha}+1\right)^{-\beta}\right]^{b}\right\}^{\theta}=\sum_{k=0}^{\infty} a_{k}\left\{\left[1-\left(x^{\alpha}+1\right)^{-\beta}\right]^{b}\right\}^{k}
$$

where:

$$
a_{k}=(-1)^{k}\left(\begin{array}{l}
\theta \\
k
\end{array}\right)
$$

Now, applying (5) for $1-\log \left\{1-\left[1-\left(x^{\alpha}+1\right)^{-\beta}\right]^{b}\right\}$ still in Equation (2), we obtain:

$$
\begin{aligned}
& 1-\log \left\{1-\left[1-\left(x^{\alpha}+1\right)^{-\beta}\right]^{b}\right\} \\
& =1+\sum_{i=0}^{\infty}\left\{\left\{\left[1-\left(x^{\alpha}+1\right)^{-\beta}\right]^{b}\right\}^{i+1} /(i+1)\right) \\
& =\sum_{k=0}^{\infty} b_{k}\left\{\left[1-\left(x^{\alpha}+1\right)^{-\beta}\right]^{b}\right\}^{k},
\end{aligned}
$$

where, $b_{0}=1$ and:

$$
\text { for } k \geq 1, b_{k}=\frac{-1}{k}
$$

Then, Equation (2) can be written as:

$$
\begin{aligned}
& F_{\theta, b, \alpha, \beta}(x)=1-\frac{\sum_{k=0}^{\infty} a_{k}\left\{\left[1-\left(x^{\alpha}+1\right)^{-\beta}\right]^{b}\right\}^{k}}{\sum_{k=0}^{\infty} b_{k}\left\{\left[1-\left(x^{\alpha}+1\right)^{-\beta}\right]^{b}\right\}^{k}} \\
& =1-\sum_{k=0}^{\infty} c_{k}\left\{\left[1-\left(x^{\alpha}+1\right)^{-\beta}\right]^{b}\right\}^{k},
\end{aligned}
$$

where, $c_{0}=\frac{a_{o}}{b_{o}}$ and, for $k \geq 1$, we have:

$$
c_{k}=\frac{1}{b_{0}}\left(a_{k}-\frac{1}{b_{0}} \sum_{r=1}^{k} b_{r} c_{k-r}\right) .
$$


At the end, the CDF (2) can be written as:

$$
\begin{aligned}
& F_{\theta, b, \alpha, \beta}(x)=\sum_{k=0}^{\infty} d_{k+1} \prod_{k+1}(x) \\
& =\sum_{k=0}^{\infty} d_{k+1}\left\{\left[1-\left(x^{\alpha}+1\right)^{-\beta}\right]^{b}\right\}^{k+1},
\end{aligned}
$$

where, $d_{0}=1-c_{k}$, for $k \geq 1$ we have $d_{0}=-c_{k}$ and:

$$
\prod_{k+1, \alpha, \beta}(x)=\left\{\left[1-\left(x^{\alpha}+1\right)^{-\beta}\right]^{b}\right\}^{k+1}
$$

is the CDF of the EBXII. By differentiating (6), we obtain the same mixture representation:

$$
f_{\theta, b, \alpha, \beta}(x)=\sum_{k=0}^{\infty} d_{k+1} \pi_{k+1, \alpha, \beta}(x)
$$

where:

$$
\begin{aligned}
& \pi_{k+1, \alpha, \beta}(x)=b(k+1) \alpha \beta x^{\alpha-1}\left(x^{\alpha}+1\right)^{-\beta-1} \\
& {\left[1-\left(x^{\alpha}+1\right)^{-\beta}\right]^{b-1}\left\{\left[1-\left(x^{\alpha}+1\right)^{-\beta}\right]^{b}\right\}^{k}}
\end{aligned}
$$

is the PDF of the EBXII. Using (4) the last expression can be rewritten as:

$$
f_{\theta, b, \alpha, \beta}(x)=\sum_{r=0}^{\infty} d_{r} g_{\alpha, \beta(1+r)}(x),
$$

where:

$$
g_{\alpha, \beta(1+r)}(x)=\alpha \beta(1+r) x^{\alpha-1}\left(x^{\alpha}+1\right)^{-\beta(1+r)-1}
$$

is the BXII density with parameters $\alpha$ and $\beta(1+r)$ and:

$$
d_{r}=\sum_{k=0}^{\infty} d_{k+1} \frac{(-1)^{r}}{1+r} b(k+1)\left(\begin{array}{c}
-1+b(k+1) \\
r
\end{array}\right)
$$

Equation (7) reveals that the BHEBXII density function is a linear combination of the EBXIIdensity. Thus, some structural properties of the new family such as the ordinary and incompletemoments and generating function can be immediately obtained from wellestablished propertiesof the EBXIID.

\section{Moments}

The $r^{\text {th }}$ ordinary moment of $X$ is given by:

$$
\mu_{n}^{\prime}=E\left(X^{r}\right)=\int_{-\infty}^{\infty} x^{n} f_{\theta, b, \alpha, \beta}(x) d x
$$

Then, we obtain:

$$
\mu_{n}^{\prime}=\left.\sum_{r=0}^{\infty} d_{r} \beta(1+r) B\left(\beta(1+r)-n \alpha^{-1}, n \alpha^{-1}+1\right)\right|_{(n<\alpha \beta(1+r))} .
$$

Setting $n=1,2,3$ and 4 in (8), we have:

$$
\begin{aligned}
& E(X)=\mu_{1}^{\prime}=\left.\sum_{r=0}^{\infty} d_{r} \beta(1+r) B\left(\beta(1+r)-\alpha^{-1}, \alpha^{-1}+1\right)\right|_{(1<\alpha \beta(1+r))}, \\
& E\left(X^{2}\right)=\mu_{2}^{\prime}=\left.\sum_{r=0}^{\infty} d_{r} \beta(1+r) B\left(\beta(1+r)-2 \alpha^{-1}, 2 \alpha^{-1}+1\right)\right|_{(2<\alpha \beta(1+r))}, \\
& E\left(X^{3}\right)=\mu_{3}^{\prime}=\left.\sum_{r=0}^{\infty} d_{r} \beta(1+r) B\left(\beta(1+r)-3 \alpha^{-1}, 3 \alpha^{-1}+1\right)\right|_{(3<\alpha \beta(1+r))}
\end{aligned}
$$

and:

$$
E\left(X^{4}\right)=\mu_{4}^{\prime}=\left.\sum_{r=0}^{\infty} d_{r} \beta(1+r) B\left(\beta(1+r)-4 \alpha^{-1}, 4 \alpha^{-1}+1\right)\right|_{4<\alpha \beta(1+r)} .
$$

The last results can be computed numerically for most parent distributions. The skewness andkurtosis measures can be calculated from the ordinary moments using well-known relationships.

\section{Incomplete Moments}

The $n^{\text {th }}$ incomplete moment of $X$ is defined by:

$$
\tau_{n}(t)=\int_{-\infty}^{r} x^{n} f_{\theta, b, \alpha, \beta}(x) d x .
$$

We can write from (7):

$$
\tau_{n}(t)=\left.\sum_{r=0}^{\infty} d_{r} \beta(1+r) B\left(t^{\alpha} ; \beta(1+r)-n \alpha^{-1}, n \alpha^{-1}+1\right)\right|_{(n<\alpha \beta(1+r))},
$$

where:

$$
B(a, b)=\int_{0}^{\infty}(1+t)^{-(a+b)} t^{a-1} d t,
$$

and:

$$
B(p ; a, b)=\int_{0}^{p}(1+t)^{-(a+b)} t^{a-1} d t
$$

are the beta and the incomplete beta functions of the second type respectively. Setting $n=1$ and 4 in (9), we have:

$$
\tau_{1}(t)=\left.\sum_{r=0}^{\theta} d_{r} \beta(1+r) B\left(t^{a} ; \beta(1+r)-\alpha^{-1}, \alpha^{-1}+1\right)\right|_{(1<\alpha \beta(1+r))},
$$

which is the first incomplete moment.

\section{Moment Generating Function}

The Moment Generating Function (MGF) of $X$, say $M_{X}(t)=E[\exp (t X)]$, can be obtained from (7) as: 


$$
M_{X}(t)=\sum_{r=0}^{\infty} d_{r} M_{r}(t)
$$

where, $M_{r}(t)$ is the MGF of the BXIID with parameters $\alpha, \beta(1+r)$. However, Paranaíba et al. (2011) provided a simple representation for the MGF of the BXIID. In a similar manner, weprovide another representation for the MGF, say $M_{X}(t)$, of the $\operatorname{BXII}(\alpha, \beta)$ model. For $t<0$, wecan write:

$$
M(t)=\alpha \beta \int_{0}^{\infty} \exp (y t) y^{\alpha-1}\left(1+y^{\alpha}\right)^{-\beta-1} d y
$$

Next, we require the Meijer G-function defined by:

$$
\begin{aligned}
& G_{[p, q]}^{[m, n]}\left(x \mid \begin{array}{c}
a_{1}, \ldots, a_{p} \\
b_{1}, \ldots, b_{q}
\end{array}\right) \\
& =\frac{1}{2 \pi i} \int \frac{\prod_{L=1}^{m} \Gamma\left(b_{j}+t\right) \prod_{j=1}^{n} \Gamma\left(1-a_{j}+t\right)}{\prod_{j=n+1}^{p} \Gamma\left(a_{j}+t\right) \prod_{j=m+1}^{p} \Gamma\left(1-b_{j}-t\right)} x^{-t} d t,
\end{aligned}
$$

where, $i=\sqrt{-1}$ is the complex unit and $L$ denotes an integration path (Gradshteyn and Ryzhik, 2000). The Meijer G-function contains as particular cases many integrals with elementary and special functions (Prudnikov et al., 1986). We now assume that $\alpha=m / \beta$, where $m$ and $\beta$ are positive integers. This condition is not restrictive since every positive real number can beapproximated by a rational number. We have the following result, which holds for $m$ and $k$ positive integers, $\mu>-1$ and $p>0$ (Prudnikov et al., 1992):

$$
\begin{aligned}
& \left.I\left(p, \mu, \frac{m}{\beta}, u\right)\right|_{0} ^{\infty}=\int_{0}^{\infty} \exp (-p x) x^{\mu}\left(1+x^{m / \beta}\right)^{u} d x \\
& =G_{[\beta+m, \beta]}^{[\beta, \beta+m]}\left(\left.\left(m^{m} p^{-m}\right)\right|^{\Delta(m,-\mu), \Delta(\beta, u+1)}\right) \\
& \times\left[(2 \pi)^{\frac{m-1}{2}} p^{\mu+1} \Gamma(-u)\right]^{-1}\left(\beta^{-u} m^{\mu+\frac{1}{2}}\right),
\end{aligned}
$$

where:

$$
\Delta(\tau, \zeta)=\zeta / \tau,(\zeta+1) / \tau, \ldots,(\zeta+\tau) / \tau
$$

We can write (for $t<0$ ):

$$
M(t)=m I\left(-t, \frac{m}{\beta}-1, \frac{m}{\beta},-\beta-1\right) .
$$

Hence, the MGF of $X$ can be expressed as:

$$
M_{X}(t)=\left.m \sum_{r=0}^{\infty} d_{r}\left[I\left(\begin{array}{l}
-t,-1+\frac{m}{\beta(1+r)}, \frac{m}{\beta(1+r)}, \\
-[\beta(1+r)+1]
\end{array}\right)\right]\right|_{0} ^{\infty} .
$$

\section{Moment of Residual Life and Reversed Residual} Life

The $n^{\text {th }}$ moment of the residual life, denoted by:

$$
m_{n}(t)=\left.E\left[(X-t)^{n}\right]\right|_{(X>t, n=1,2, \ldots)},
$$

The $n^{\text {th }}$ moment of the residual life of $X$ is given by:

$$
m_{n}(t)=\frac{\int_{t}^{\infty}(x-t)^{n} d F_{\theta, b, \alpha, \beta}(x)}{1-F(t)} .
$$

Then, we can write:

$$
\begin{aligned}
& m_{n}(t)=\frac{1}{1-F(t)} \sum_{i=0}^{n} \sum_{r=0}^{\infty} \frac{(-1)^{n-i} n ! t^{n-i}}{i ! \Gamma(n-i+1)} d_{r} \beta(1+r) \\
& B\left(t^{\alpha} ; \beta(1+r)-n \alpha^{-1}, n \alpha^{-1}+1\right) .
\end{aligned}
$$

Another interesting function is the Mean Residual Life (MRL) function or the life expectation atage $x$ defined by $m_{1}(t)=\left.E[(X-t)]\right|_{(X>t, n=1)}$, which represents the expected additional life length for a unit which is alive at age $x$. The MRL of the WBXII distribution can be obtained by setting $n=1$ in the last equation. The $n^{\text {th }}$ moment of the reversed residual life, say:

$$
M_{n}(t)=\left.E\left[(t-X)^{n}\right]\right|_{(X \leq t, t>0, n=1,2, \ldots)}
$$

Then, $M_{n}(t)$ is defined by:

$$
M_{n}(t)=\frac{1}{F(t)} \int_{0}^{t}(t-x)^{n} d F_{\theta, b, \alpha, \beta}(x) .
$$

The $n^{\text {th }}$ moment of the reversed residual life of $X$ :

$$
\begin{aligned}
& M_{n}(t)=\frac{1}{F(t)} \sum_{i=0}^{n} \sum_{r=0}^{\infty} \frac{(-1)^{i} n !}{i !(n-i) !} d_{r} \beta(1+r) \\
& B\left(t^{\alpha} ; \beta(1+r)-n \alpha^{-1}, n \alpha^{-1}+1\right) .
\end{aligned}
$$

The Mean Inactivity Time (MIT) or Mean Waiting Time (MWT), also called the mean reversedresidual life function, say $M_{1}(t)=\left.E[(t-X)]\right|_{(X \leq t)}$ represents the waiting time elapsed since thefailure of an item on condition that this failure had occurred in $(0, x)$. The MIT of $X$ can beobtained by setting $n=1$ in the above equation.

\section{Order Statistics}

Order statistics make their appearance in many areas of statistical theory and practice. Suppose $X_{1: n}, X_{2: n}, \ldots$, $X_{n: n}$, is a random sample from any BHEBXIID. Let $X_{i: n}$ 
denote the $i^{\text {th }}$ orderstatistic. The PDF of $X_{i: n}$ can be expressed as:

$$
f^{(i: n)}(x)=\frac{f(x)}{B(i, n-i+1)} \sum_{j=0}^{n-i}(-1)^{j}\left(\begin{array}{c}
n-i \\
j
\end{array}\right) F_{\theta, b, \alpha, \beta}(x)^{j+i-1} .
$$

We use the result 0.314 of Gradshteyn and Ryzhik (2000) for a power series raised to a positiveinteger $n$ (for $n \geq 1$ ):

$$
\left(\sum_{i=0}^{\infty} a_{i} u^{i}\right)^{n}=\sum_{i=0}^{\infty} c_{n, i} u^{i}
$$

where the coefficients $c_{n, i} \mid(i=1,2, \ldots)$ are determined from the recurrence Equation (with $c_{n, 0}=a_{0}^{n}$ ):

$$
c_{n, i}=\left(i a_{0}\right)^{-1} \sum_{m=1}^{i}[m(n+1)-i] a_{m} c_{n, i-m} .
$$

We can demonstrate that the density function of the $i^{\text {th }}$ order statistic of any BHEBXIID can beexpressed as:

$$
f_{\theta, b, \alpha, \beta}^{(i, n)}(x)=\sum_{h, k=0}^{\infty} a_{h, k} \pi_{h+k+1, \alpha, \beta}(x)=\sum_{r=0}^{\infty} d_{r}^{*} g_{\alpha, \beta(1+r)}(x),
$$

where:

$$
\begin{aligned}
& \pi_{h+k+1, \alpha, \beta}(x)=b(h+k+1) \alpha \beta x^{\alpha-1}\left(x^{\alpha}+1\right)^{-\beta-1} \\
& {\left[1-\left(x^{\alpha}+1\right)^{-\beta}\right]^{b-1}\left\{\left[1-\left(x^{\alpha}+1\right)^{-\beta}\right]^{b}\right\}^{h+k}}
\end{aligned}
$$

denotes the EBXII density function with parameter $(h+$ $k+1)$ :

$$
\begin{aligned}
& d_{r}^{*}=\sum_{k=0}^{\infty} b_{h, k} \frac{(-1)^{r}}{1+r} b(k+1)\left(\begin{array}{c}
b(h+k+1)-1 \\
r
\end{array}\right), \\
& a_{h, k}=\frac{n !(h+1)(i-1) ! d_{h}}{(h+k+1)} \sum_{j=0}^{n-i} \frac{(-1)^{j} f_{j+i-1, k}}{(n-i-j) ! j !}
\end{aligned}
$$

and $d_{h}$ is given in subsection 3.2 and the quantities $f_{j+i-}$ $1, k$ can be determined with $f_{j+i-1,0}=d_{0}^{j+i-1}$ and recursively for $k \geq 1$ :

$$
f_{j+i-1, k}=\left(k d_{0}\right)^{-1} \sum_{m=1}^{k}[m(j+i)-k] d_{m} f_{j+i-1, k-m} .
$$

Using (10) we have:

$$
E\left(X_{i: n}^{q}\right)=\sum_{r=0}^{\infty} d_{r}^{*} \beta(1+r) B\left(\beta(1+r)-\frac{q}{\alpha}, \frac{q}{\alpha}+1\right) \mathrm{I}_{(q<\alpha \beta(1+r))} .
$$

\section{Estimation}

Several approaches for parameter estimation were proposed in the literature but the maximum likelihood method is the most commonly employed. The Maximum Likelihood Estimators (MLEs) enjoy desirable properties and can be used for constructing confidence intervals and regions and in test statistics. The normal approximation for these estimators in large samples can beeasily handled either analytically or numerically. So, we consider the estimation of the unknownparameters of this model from complete samples only by maximum likelihood. Let $x_{1}, \ldots, x_{n}$ be a random sample from the BHEBXII distribution with parameters $\theta, b, \alpha$ and $\beta$. Let $\Upsilon=(\theta, b, \alpha, \beta)^{T}$ be the $4 \times 1$ parameter vector. For determining the MLE of $\Upsilon$, we have the log-likelihood function:

$$
\begin{aligned}
& \ell=\ell(\Upsilon)=n \log b+n \log \alpha+n \log \beta \\
& +(\alpha-1) \sum_{i=1}^{n} \log x_{i}-(\beta+1) \sum_{i=1}^{n} \log \left(x_{i}^{\alpha}+1\right) \\
& -2 \sum_{i=1}^{n} \log \left(1-\log \left\{1-\left[1-\left(x_{i}^{\alpha}+1\right)^{-\beta}\right]^{b}\right\}\right) \\
& +\sum_{i=1}^{n} \log \left\{\theta\left(1-\log \left\{1-\left[1-\left(x_{i}^{\alpha}+1\right)^{-\beta}\right]^{b}\right\}\right)+1\right\} \\
& +(b-1) \sum_{i=1}^{n} \log \left[1-\left(x_{i}^{\alpha}+1\right)^{-\beta}\right]+(\theta-1) \sum_{i=1}^{n} \log \left\{1-\left[1-\left(x_{i}^{\alpha}+1\right)^{-\beta}\right]^{b}\right\} .
\end{aligned}
$$

The components of the score vector, $L(\Upsilon)=$ $\frac{\partial \ell}{\partial \Upsilon}=\left(\frac{\partial \ell}{\partial \theta}, \frac{\partial \ell}{\partial b}, \frac{\partial \ell}{\partial \epsilon}, \frac{\partial \ell}{\partial \beta},\right)^{T}$ are available if needed. Set-ting the nonlinear system of equations $L_{\theta}=L_{b}=L_{\alpha}=L_{\beta}=0$ and solving them simultaneously yields the MLE $\hat{\Upsilon}=(\hat{\theta}, \hat{b}, \hat{\alpha}, \hat{\beta})^{T}$

\section{Simulation Study}

We simulate the new model by taking $n=20,50$, 150, 300, 500 and 1000. For each sample size, weevaluate the ML Estimations (MLEs) of the parameters using the optim function of the $R$ software.Then, we repeat this process 1000 times and compute the Averages of the Estimates (AEs) and Mean Squared Errors (MSEs). Table 1 gives all simulation results. The values in Table 1 indicatethat the MSEs of $\hat{\theta}, \hat{b}, \hat{\alpha}$ and $\hat{\beta}$ decay toward zero when $\mathrm{n}$ increases for all settings of $\theta, b, \alpha$ and $\beta$ as expected under first-under asymptotic theory. The AEs of the parameters tend to be closerto the true parameter values when $n$ increases. This fact supports that the asymptotic normaldistribution provides an adequate approximation to the finite sample distribution of the MLEs. 
Table 1: The AEs and MSEs based on 1000 simulations

\begin{tabular}{lllllll}
\hline Parameters & 20 & 50 & 150 & 300 & 500 & 1000 \\
\hline$\theta=1.5$ & 1.514567 & 1.520876 & 1.512251 & 1.501886 & 1.506182 & 1.499782 \\
& $(0.3521752)$ & $(0.1441626)$ & $(0.0442235)$ & $(0.0195935)$ & $(0.012842)$ & $(0.0059777)$ \\
$\alpha=0.6$ & 0.616176 & 0.606417 & 0.600229 & 0.599789 & 0.601936 & 0.599593 \\
& $(0.0151023)$ & $(0.004884)$ & $(0.0015242)$ & $(0.0007819)$ & $(0.0004321)$ & $(0.0002444)$ \\
$b=0.9$ & 0.927605 & 0.911668 & 0.900743 & 0.902986 & 0.900571 & 0.901248 \\
& $(0.0255461)$ & $(0.0097934)$ & $(0.003195)$ & $(0.0016938)$ & $(0.0008961)$ & $(0.0004464)$ \\
$\beta=0.8$ & 0.825944 & 0.825254 & 0.80551 & 0.801926 & 0.80257 & 0.800766 \\
& $(0.0787816)$ & $(0.0305656)$ & $(0.003195)$ & $(0.0038182)$ & $(0.0022859)$ & $(0.0010038)$ \\
$\theta=2$ & 2.021285 & 2.028191 & 2.000062 & 2.003788 & 1.992041 & 1.999274 \\
& $(0.5457159)$ & $(0.2184421)$ & $(0.0639926)$ & $(0.0639926)$ & $(0.0178037)$ & $(0.0093023)$ \\
$\alpha=0.7$ & 0.718024 & 0.703211 & 0.698492 & 0.701874 & 0.700009 & 0.700604 \\
& $(0.018831)$ & $(0.0058359)$ & $(0.0018581)$ & $(0.0009923)$ & $(0.0005863)$ & $(0.0002953)$ \\
$b=1.1$ & 0.42716924 & 1.104061 & 1.103025 & 1.101393 & 1.099822 & 1.100314 \\
& $(0.0301518)$ & $(0.0113167)$ & $(0.003931)$ & $(0.0018997)$ & $(0.0012092)$ & $(0.0005613)$ \\
$\beta=0.9$ & 0.92589 & 0.908232 & 0.905625 & 0.903091 & 0.898238 & 0.901076 \\
& $(0.0711364)$ & $(0.0245606)$ & $(0.0075746)$ & $(0.0037931)$ & $(0.0019012)$ & $(0.0010895)$ \\
$\theta=2.5$ & 2.549126 & 2.549466 & 2.498054 & 2.50238 & 2.485818 & 2.503147 \\
& $(1.0390537)$ & $(0.4208019)$ & $(0.1289025)$ & $(0.0711908)$ & $(0.0396652)$ & $(0.01908)$ \\
$\alpha=0.9$ & 0.92072 & 0.90809 & 0.89835 & 0.903032 & 0.900493 & 0.900111 \\
& $(0.0195577)$ & $(0.0070849)$ & $(0.0021345)$ & $(0.0011709)$ & $(0.0006917)$ & $(0.0003626)$ \\
$b=1.4$ & 1.431182 & 1.411168 & 1.405789 & 1.401176 & 1.404374 & 1.400468 \\
& $(0.0428515)$ & $(0.0167855)$ & $(0.0049324)$ & $(0.0027645)$ & $(0.001501)$ & $(0.0007583)$ \\
$\beta=1.5$ & 1.54377 & 1.531398 & 1.516941 & 1.500401 & 1.503514 & 1.500571 \\
& $(0.153552)$ & $(0.059333)$ & $(0.017966)$ & $(0.009102)$ & $(0.0054631)$ & $(0.002784)$ \\
\hline & & & & & &
\end{tabular}

\section{Applications}

In this section, we provide two applications to real data sets to illustrate the importance andpotentiality of the BHEBXIID. For these data, we compare the BHEBXIID, with beta BurrXII (BXIID), Marshall-Olkin BurrXII (MOBXIID), Topp Leone Burr XII (TLBXIID), Kumaraswamy BurrXII (KwBXIID), BBXIID, beta exponentiated BurrXII (BEBXIID), Five parameter beta BurrXII (FBBXIID), Five parameter Kumaraswamy BurrXII (FKwBXIID) and Zografos-Balakrishnan BurrXII (ZBBXIID) (see the PDFs in Appendix A).

Data Set I: Breaking stress data. This data set consists of 100 observations of breaking stress of carbon fibres (in Gba) given by Nichols and Padgett (2006). Data Set II: Taxes revenue data.The actual taxes revenue data (in 1000 million Egyptian pounds). This data set were used by Nassar and Nada (2011) and Yousof et al. (2015) (see the data sets in Appendix B).

The Total Time Test (TTT) plot due to Aarset (1987) is an important graphical approach to verify whether the data can be applied to a specific distribution or not. According to Aarset (1987), theempirical version of the TTT plot is given by plotting:

$$
T(r / n)=\left[\sum_{i=1}^{r} y_{i: n}+(n-r) y_{r: n}\right] / \sum_{i=1}^{r} y_{i: n}
$$

against $r / n$, where $r=1, \ldots, n$ and $y_{i: n}(i=1, \ldots, n)$ are the order statistics of the sample. Aarset (1987) showed that the HRF is constant if the TTT plot is graphically presented as a straightdiagonal, the HRF is increasing (or decreasing) if the TTT plot is concave (or convex). The HRFis U-shaped (bathtub) if the TTT plot is firstly convex and then concave, if not, the HRF is unimodal. The TTT plots the three real data sets is presented in Fig. 3 and 4. This plotindicates that the empirical HRFs of the two data sets are increasing.

In order to compare the fitted models, we consider the following goodness-of-fit statistics: The Akaike Information Criterion (AIC), Bayesian Information Criterion (BIC), Hannan-Quinn Information Criterion (HQIC), Consistent Akaike Information Criterion (CAIC), where:

$$
\begin{aligned}
& A I C=2[-\ell(\hat{\Upsilon})+k], \\
& B I C=2\left[-\ell(\hat{\Upsilon})+\frac{1}{2} k \log (n)\right], \\
& H Q I C=2\{-\ell(\hat{\Upsilon})+k \log (n)[\log (n)]\}
\end{aligned}
$$

and:

$$
C A I C=2[-\ell(\hat{\Upsilon})+k n /(n-k-1)],
$$

where, $k$ is the number of parameters, $\mathrm{n}$ is the sample size, $-2 \ell(\hat{\Upsilon})$ is the maximized log-likelihood. Generally, the smaller these statistics are, the better the fit. 

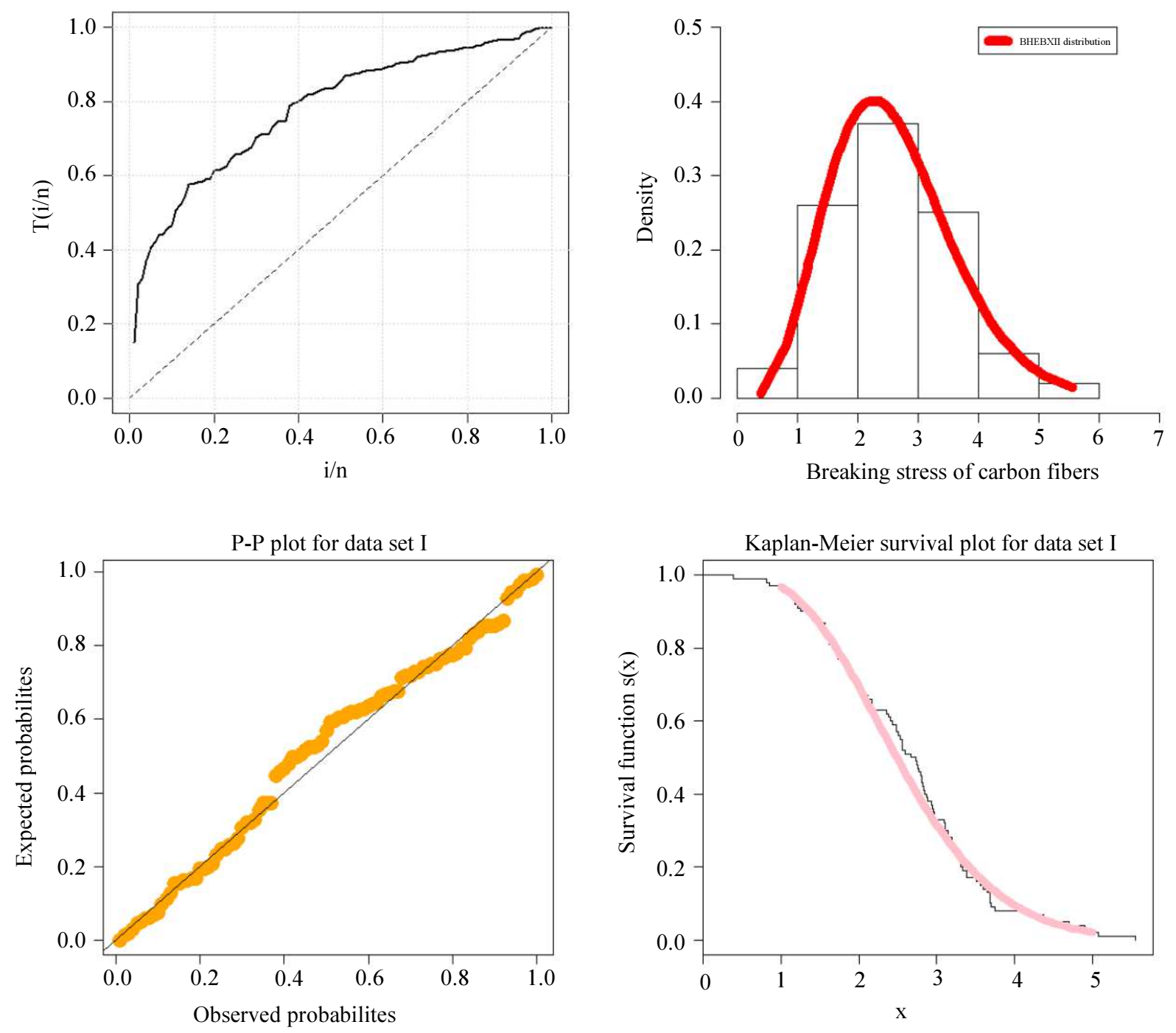

Fig. 3: TTT plot, histogram, P-P plot, Kaplan-Meier survival for data set I
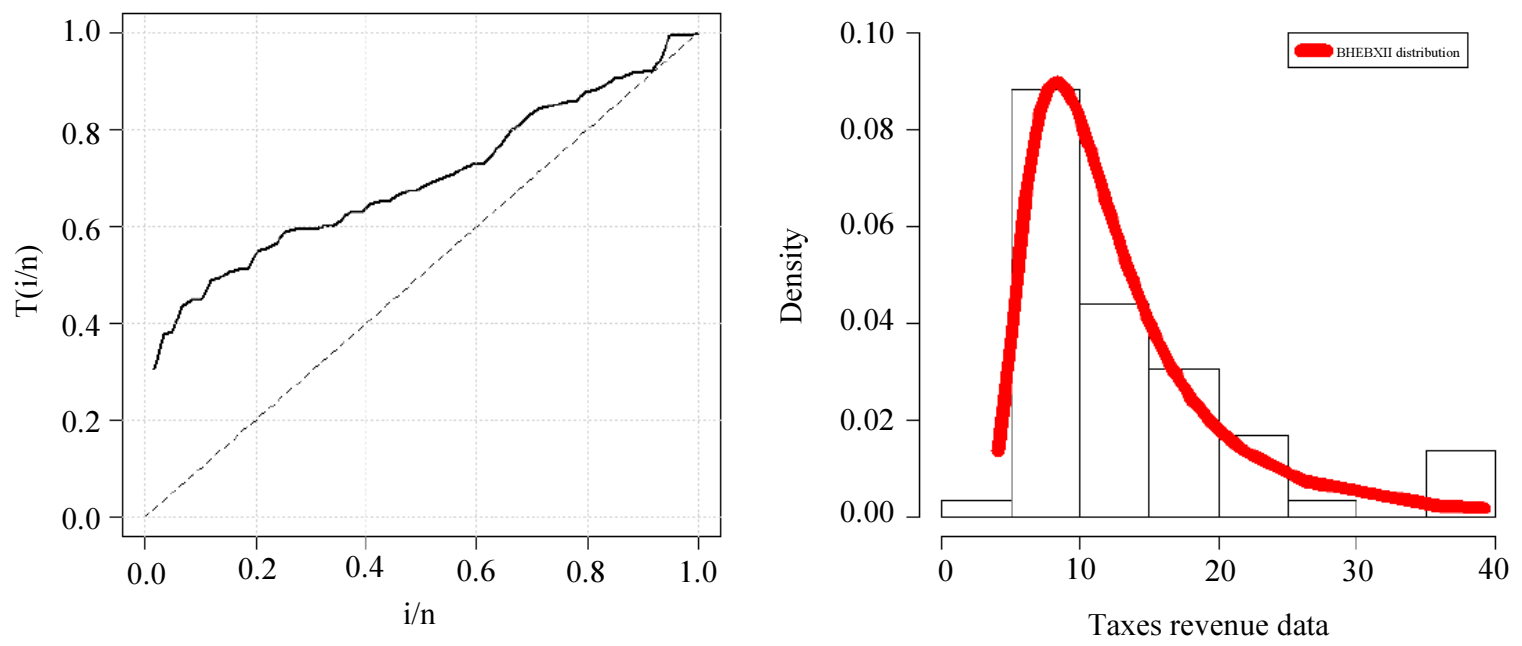

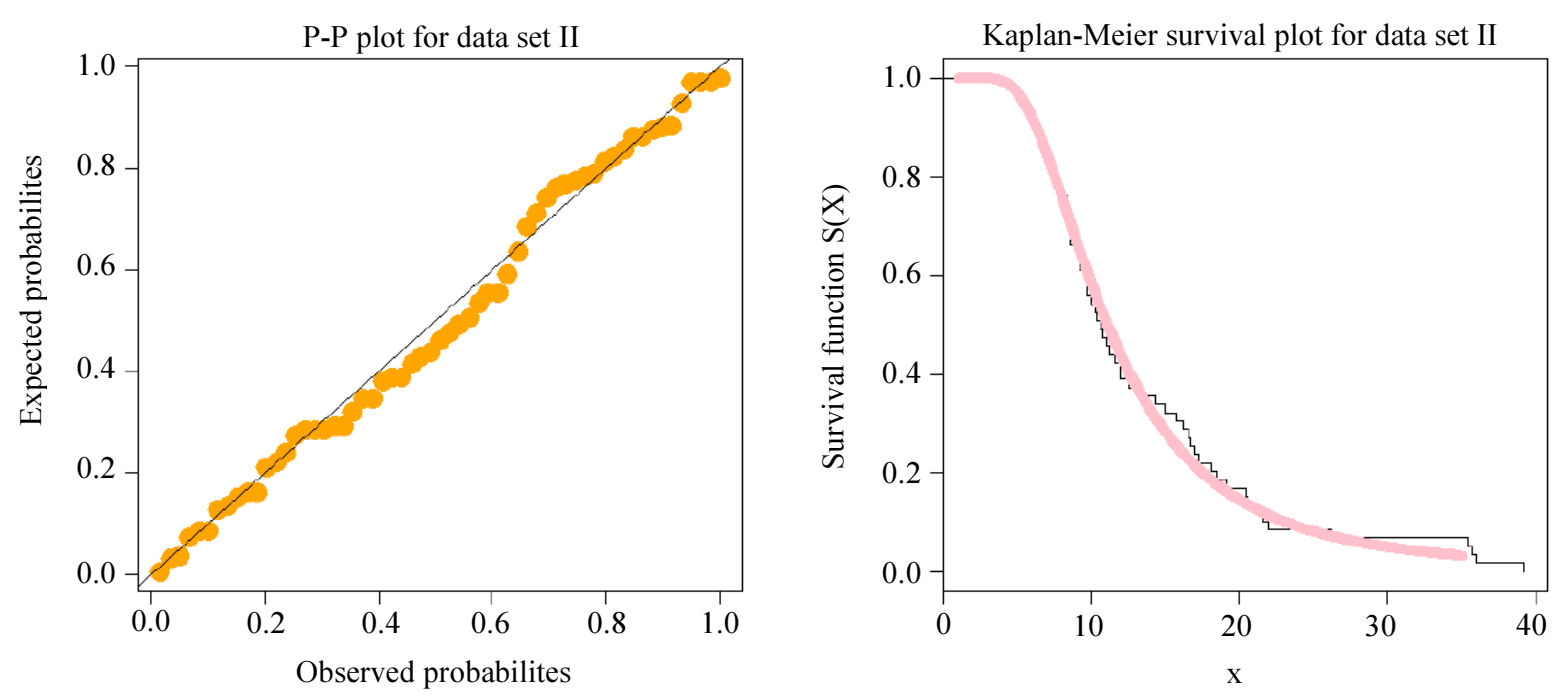

Fig. 4: TTT plot, histogram, P-P plot, Kaplan-Meier survival for data set II

Table 2: MLEs and standard errors, confidence interval (in parentheses) for the data set I

\begin{tabular}{|c|c|c|c|c|c|}
\hline Model & $\hat{a}$ & $\hat{b}$ & $\hat{\alpha}$ & $\hat{\beta}$ & $\hat{\theta}$ \\
\hline \multirow[t]{3}{*}{ BXIID } & - & - & 5.941 & 0.187 & - \\
\hline & - & - & $(1.279)$ & $(0.044)$ & - \\
\hline & - & - & $(3.43,8.45)$ & $(0.10,0.27)$ & - \\
\hline \multirow[t]{3}{*}{ MOBXIID } & - & - & 1.192 & 4.834 & 838.73 \\
\hline & - & - & $(0.952)$ & $(4.896)$ & $(229.34)$ \\
\hline & - & - & $(0,3.06)$ & $(0,14.43)$ & $(389.22,1288.24)$ \\
\hline \multirow[t]{3}{*}{ TLBXIID } & - & - & 1.350 & 1.061 & 13.728 \\
\hline & - & - & $(0.378)$ & $(0.384)$ & $(8.400)$ \\
\hline & - & - & $(0.61,2.09)$ & $(0.31,1.81)$ & $(0,30.19)$ \\
\hline \multirow[t]{3}{*}{ KwBXIID } & 48.103 & 79.516 & 0.351 & 2.730 & - \\
\hline & $(19.348)$ & $(58.186)$ & $(0.098)$ & (1.077) & - \\
\hline & $(10.18,86.03)$ & $(0,193.56)$ & $(0.16,0.54)$ & $(0.62,4.84)$ & - \\
\hline \multirow[t]{3}{*}{ BBXIID } & 359.683 & 260.097 & 0.175 & 1.123 & - \\
\hline & $(57.941)$ & (132.213) & $(0.013)$ & $(0.243)$ & - \\
\hline & $(246.1,473.2)$ & $(0.96,519.2)$ & $(0.14,0.20)$ & $(0.65,1.6)$ & - \\
\hline \multirow[t]{3}{*}{ BEBXIID } & 0.381 & 11.949 & 0.937 & 33.402 & 1.705 \\
\hline & $(0.078)$ & $(4.635)$ & $(0.267)$ & $(6.287)$ & $(0.478)$ \\
\hline & $(0.23,0.53)$ & $(2.86,21)$ & $(0.41,1.5)$ & $(21,45)$ & $(0.8,2.6)$ \\
\hline \multirow[t]{3}{*}{ FBBXII } & 0.421 & 0.834 & 6.111 & 1.674 & 3.450 \\
\hline & $(0.011)$ & $(0.943)$ & $(2.314)$ & $(0.226)$ & (1.957) \\
\hline & $(0.4,0.44)$ & $(0.2 .7)$ & $(1.57,10.7)$ & $(1.23,2.1)$ & $(0,7)$ \\
\hline \multirow[t]{3}{*}{ FKwB-XII } & 0.542 & 4.223 & 5.313 & 0.411 & 4.152 \\
\hline & $(0.137)$ & $(1.882)$ & $(2.318)$ & $(0.497)$ & (1.995) \\
\hline & $(0.3,0.8)$ & $(0.53,7.9)$ & $(0.9,9)$ & $(0,1.7)$ & $(0.2,8)$ \\
\hline \multirow[t]{3}{*}{ ZBB-XII } & 123.101 & - & 0.368 & 139.247 & - \\
\hline & $(243.011)$ & - & $(0.343)$ & $(318.546)$ & - \\
\hline & $(0,599.40)$ & - & $(0,1.04)$ & $(0,763.59)$ & - \\
\hline \multirow[t]{3}{*}{ BHEBXIID } & - & 33.23 & 0.369 & 2.122 & 193.71 \\
\hline & - & $(0.000)$ & $(0.069)$ & $(0.505)$ & $(13.36)$ \\
\hline & - & - & $(0,0.505)$ & $(0,3.8)$ & $(166.15,219,8)$ \\
\hline
\end{tabular}

Based on the values in Table 2-5 the BHEBXIID provides adequate fits as compared to BXIID, MOBXIID, TLBXIID, KwBXIID, BBXIID, BEBXIID, FBBXIID, FKwBXIID and ZBBXIID in application with small values for AIC, BIC, CAIC and HQIC. From our findings it is seen that in the applications cases considered here the proposed BHEBXIID turned out to be the best model in terms 
of different selection criteria. Moreover, from the plots of estimated PDF against the observed histograms reveals that the new distribution provides closest fit to all the data sets. It may be mentioned that the new distribution has even outperformed the four and five parameter extensions considered the two applications. It is therefore is a useful contribution to the existing set of extended BXIID.

Table 3: AIC, BIC, CAIC and HQIC values for the data set I

\begin{tabular}{lllll}
\hline Model & AIC & BIC & CAIC & HQIC \\
\hline BXIID & 382.94 & 388.15 & 383.06 & 385.05 \\
MOBXIID & 305.78 & 313.61 & 306.03 & 308.96 \\
TLBXIID & 323.52 & 331.35 & 323.77 & 326.70 \\
KwBXIID & 303.76 & 314.20 & 304.18 & 308.00 \\
BBXIID & 305.64 & 316.06 & 306.06 & 309.85 \\
BEBXIID & 305.82 & 318.84 & 306.46 & 311.09 \\
FBBXII & 304.26 & 317.31 & 304.89 & 309.56 \\
FKwB-XII & 305.50 & 318.55 & 306.14 & 310.80 \\
ZBB-XII & 302.96 & 310.78 & 303.21 & 306.13 \\
BHEBXIID & 292.58 & 303.00 & 293.004 & 296.80 \\
\hline
\end{tabular}

Table 4: MLEs and standard errors, confidence interval (in parentheses) for the data set II

\begin{tabular}{|c|c|c|c|c|c|}
\hline Model & $\hat{a}$ & $\hat{b}$ & $\hat{\alpha}$ & $\hat{\beta}$ & $\hat{\theta}$ \\
\hline \multirow[t]{3}{*}{$\overline{\text { BXIID }}$} & - & - & 5.615 & 0.072 & - \\
\hline & - & - & $(15.048)$ & $(0.194)$ & - \\
\hline & - & - & $(0,35.11)$ & $(0,0.45)$ & - \\
\hline \multirow[t]{3}{*}{ MOBXIID } & - & - & 8.017 & 0.419 & 70.359 \\
\hline & - & - & $(22.083)$ & $(0.312)$ & $(63.831)$ \\
\hline & - & - & $(51.29)$ & $(0,1.03)$ & $(0,195.47)$ \\
\hline \multirow[t]{3}{*}{ TLBXIID } & - & - & 91.320 & 0.012 & 141.073 \\
\hline & - & - & $(15.071)$ & $(0.002)$ & $(70.028)$ \\
\hline & - & - & $(61.78,120.86)$ & $(0.008,0.02)$ & $(3.82,278.33)$ \\
\hline \multirow[t]{3}{*}{ KwBXIID } & 18.130 & 6.857 & 10.694 & 0.081 & - \\
\hline & $(3.689)$ & $(1.035)$ & $(1.166)$ & $(0.012)$ & - \\
\hline & $(10.89,25.36)$ & $(4.83,8.89)$ & $(8.41,12.98)$ & $(0.06,0.10)$ & - \\
\hline \multirow[t]{3}{*}{ BBXIID } & 26.725 & 9.756 & 27.364 & 0.020 & - \\
\hline & $(9.465)$ & $(2.781)$ & $(12.351)$ & $(0.007)$ & - \\
\hline & $(8.17,45.27)$ & $(4.31,15.21)$ & $(3.16,51.57)$ & $(0.006,0.03)$ & - \\
\hline \multirow[t]{3}{*}{ BEBXIID } & 2.924 & 2.911 & 3.270 & 12.486 & 0.371 \\
\hline & $(0.564)$ & $(0.549)$ & $(1.251)$ & (6.938) & $(0.788)$ \\
\hline & $(1.82,4.03)$ & $(1.83,3.99)$ & $(0.82,5.72)$ & $(0,26.08)$ & $(0,1.92)$ \\
\hline \multirow[t]{3}{*}{ FBBXIID } & 30.441 & 0.584 & 1.089 & 5.166 & 7.862 \\
\hline & $(91.745)$ & $(1.064)$ & $(1.021)$ & $(8.268)$ & $(15.036)$ \\
\hline & $(0,210.26)$ & $(0,2.67)$ & $(0,3.09)$ & $(0,21.37)$ & $(0,37.33)$ \\
\hline \multirow[t]{3}{*}{ FKwBXIID } & 12.878 & 1.225 & 1.665 & 1.411 & 3.732 \\
\hline & $(3.442)$ & $(0.131)$ & $(0.034)$ & $(0.088)$ & $(1.172)$ \\
\hline & $(6.13,19.62)$ & $(0.97,1.48)$ & $(1.56,1.73)$ & $(1.24,1.58)$ & $(1.43,6.03)$ \\
\hline \multirow[t]{3}{*}{ BHEBXIID } & - & 34.842 & 9.84 & 0.13033 & 2.27 \\
\hline & - & $(28.8)$ & $(22.29)$ & $(0.29)$ & $(2.177)$ \\
\hline & - & $(0,89.78)$ & $(0,52.92)$ & $(0,0.7)$ & $(0,6.57)$ \\
\hline
\end{tabular}

Table 5: AIC, BIC, CAIC and HQIC values for the data set II

\begin{tabular}{lllll}
\hline Model & AIC & BIC & CAIC & HQIC \\
\hline BXIID & 518.46 & 522.62 & 518.67 & 520.080 \\
MOBXIID & 387.22 & 389.38 & 387.66 & 389.680 \\
TLBXIID & 385.94 & 392.18 & 386.38 & 388.400 \\
KwBXIID & 385.58 & 393.90 & 386.32 & 388.860 \\
BBXIID & 385.56 & 394.10 & 386.30 & 389.100 \\
BEBXIID & 387.04 & 397.42 & 388.17 & 391.090 \\
FBBXIID & 386.74 & 397.14 & 387.87 & 390.840 \\
FKwB-XIID & 386.96 & 397.36 & 388.09 & 391.060 \\
BHEBXIID & 384.82 & 393.13 & 385.56 & 388.059 \\
\hline
\end{tabular}




\section{Conclusion}

In this article, a new four parameter Burr-Hatke Exponentiated Burr XII Distribution (BHEBXIID) is defined and studied. Several structural mathematical properties of the proposed model areinvestigated. The Maximum Likelihood (ML) method is used to estimate the model parameters. We assess the performance of the MLEs of the new distribution with respect to sample size $n$. The assessment was based on a simulation study. The new distribution is applied for modelingtwo real data sets to prove its flexibility empirically. It is shown that the new lifetime model canbe viewed as a simple linear mixture of the Burr XII density. It can be viewed as a suitable model for fitting the unimodal and the right skewed data sets. The new model provides appropriate fitsas compared to other extensions of the Burr XII models by means of two real data applicationswith small values for AIC, BIC, CAIC and HQIC. Plots for the Estimated PDFs, P-P, TTT andKaplanMeier Survival are provided for the two real data sets.

\section{Acknowledgment}

The author gratefully acknowledge with thanks the very thoughtful and constructive comments and suggestions of the Editor-in-Chief and the reviewers which resulted in much improved paper.

\section{Ethics}

The author declares that there is no conflict of interests regarding the publication of this article.

\section{References}

Aarset, M.V., 1987. How to identify a bathtub hazard rate. IEEE Trans. Reliability, 36: 106-108.

Afify, A.Z., G.M. Cordeiro, E.M.M. Ortega, H.M. Yousof and N.S. Butt, 2018. The four-parameter Burr XII distribution: Properties, regression model and applications. Commun. Stat. Theory Meth., 47: 2605-2624. DOI: 10.1080/03610926.2016.1231821

Altun, E., H.M. Yousof and G.G. Hamedani, 2018a.A new log-location regression model with influence diagnostics and residual analysis. Int. J. Applied Math. Stat., 33: 417-449. DOI: 10.22190/FUMI1803417A

Altun, E., H.M. Yousof, S. Chakraborty and L. Handique, 2018b. Zografos-Balakrishnan Burr XII distribution: Regression modeling and applications. Int. J. Math. Stat.

Burr, I.W. and P.J. Cislak, 1968.On a general system of distributions I. Its curve-shaped characteristics; II. The sample median. J. Am. Stat. Assoc., 63: 627-635. DOI: 10.1080/01621459.1968.11009281

Burr, I.W., 1942. Cumulative frequency functions. Annals Math. Stat., 13: 215-232. DOI: $10.1214 / \mathrm{aoms} / 1177731607$
Burr, I.W., 1968. On a general system of distributions, III. The simple range. J. Am. Stat. Assoc., 63: 636-643.

Burr, I.W., 1973. Parameters for a general system of distributions to match a grid of 3 and 4. Commun. Stat., 2: 1-21. DOI: $10.1080 / 03610927308827052$

Cordeiro, G.M., H.M. Yousof, T.G. Ramires and E.M.M. Ortega, 2018. The Burr XII system of densities: Properties, regression model and applications. J. Stat. Comput. Simulat., 88: 432-456. DOI: $10.1080 / 00949655.2017 .1392524$

Gradshteyn, I.S. and I.M. Ryzhik, 2000. Table of Integrals, Series and Products. 6th Edn., Academic Press, San Diego.

Hatke, M.A., 1949. A certain cumulative probability function.Annals Math. Stat., 20: 461-463. DOI: $10.1214 / \mathrm{aoms} / 1177730002$

Maniu, A. I. and Voda, V. G. 2008.Generalized BurrHatke equation as generator of a homogaphic failure rate. J. Applied Quantitative Methods, 3: 215-222.

Nassar, M.M. and N.K. Nada, 2011. The beta generalized Pareto distribution. J. Stat. Adv. Theory Appl., 6: 1-17.

Nichols, M.D. and W.J. Padgett, 2006. A bootstrap control chart for Weibull percentiles. Quality Reliability Eng. Int., 22: 141-151.

DOI: $10.1002 /$ qre.691

Paranaíba, P.F.P., E.M.M. Ortega, G.M. Cordeiro and R.R. Pescim, 2011. The beta Burr XII distribution with application to lifetime data. Comput. Stat. Data Anal., 55: 1118-1136. DOI: 10.1016/j.csda.2010.09.009

Prudnikov, A.P., Y.A. Brychkov and O.I. Marichev, 1986. Integrals and series, 1. Gordon and Breach Science Publishers, Amsterdam.

Prudnikov, A.P., Y.A. Brychkov and O.I. Marichev, 1992. Integrals and Series, 4. Gordon and Breach Science Publishers, Amsterdam.

Rodriguez, R.N., 1977. A guide to the Burr type XII distributions. Biometrika, 64: 129-134.

DOI: $10.1093 /$ biomet/64.1.129

Shao, Q., 2004. Notes on maximum likelihood estimation for the three-parameter Burr XII distribution. Comput. Stat. Data Anal., 45: 675-687. DOI: $10.1016 / \mathrm{S} 0167-9473(02) 00367-5$

Silva, G.O., E.M.M. Ortega and G.A. Paula, 2010b. Residuals for $\operatorname{logBurr}$ XII regression models in survival analysis. J. Applied Stat., 38: 1435-1445. DOI: $10.1080 / 02664763.2010 .505950$

Silva, G.O., E.M.M. Ortega and G.M. Cordeiro, 2010a. The beta modified Weibull distribution. Lifetime Data Anal., 16: 409-430. DOI: 10.1007/s10985-010-9161-1

Silva, G.O., E.M.M. Ortega, V.C. Garibay and M.L. Barreto, 2008. Log-Burr XII regression models with censored data. Comput. Stat. Data Anal., 52: 3820-3842. DOI: 10.1016/j.csda.2008.01.003 
Soliman, A.A., 2005. Estimation of parameters of life from progressively censored data using Burr-XII Model. IEEE Trans. Reliability, 54: 34-42.

DOI: $10.1109 /$ TR.2004.842528

Tadikamalla, P.R., 1980. A look at the Burr and related distributions. Int. Stat. Rev., 48: 337-344.

DOI: $10.2307 / 1402945$

Wu, S.J., Y.J. Chen and C.T. Chang, 2007. Statistical inference based on progressively censored samples with random removals from the Burr type XII distribution. J. Stat. Comput. Simulat., 77: 19-27. DOI: 10.1080/10629360600569204

Yousof, H.M., A.Z. Afify, M. Alizadeh, N.S. Butt and G.G. Hamedani et al., 2015. The transmuted exponentiated generalized-G family of distributions. Pak. J. Stat. Operat. Res., 11: 441-464.

DOI: $10.18187 /$ pjsor.v11i4.1164
Yousof, H.M., E. Altun, T.G. Ramires, M. Alizadeh and M. Rasekhi, 2018a. A new family of distributions with properties, regression models and applications. J. Stat. Manage. Syst., 21: 163-188.

DOI: $10.1080 / 09720510.2017 .1411028$

Yousof, H.M., M. Rasekhi, E. Altun, M. Alizadeh and G.G. Hamedani et al., 2018b. A new lifetime model with regression models, characterizations and applications. Commun. Stat. Simulat. Comput. DOI: 10.1080/03610918.2017.1377241

Zimmer, W.J., J.B. Keats and F.K. Wang, 1998.The Burr XII distribution in reliability analysis. J. Quality Technol., 30: 386-394.

DOI: $10.1080 / 00224065.1998 .11979874$

\section{Appendix A}

In this appendix we provide the densities used in the applications:

$$
\begin{aligned}
& f_{\text {BXIID }}(x)=2 b \alpha \beta x^{\alpha-1}\left(x^{\alpha}+1\right)^{-2 \beta-1}\left[1-\left(x^{\alpha}+1\right)^{-2 \beta}\right]^{b-1} ; \\
& f_{\text {MOВХІІD }}(x)=\alpha \beta \theta x^{\alpha-1}\left(x^{\alpha}+1\right)^{-\beta-1}\left[1-(1-\theta)\left(x^{\alpha}+1\right)^{-\beta}\right]^{-2} ; \\
& f_{\text {TLBXIID }}(x)=2 \theta \alpha \beta x^{\alpha-1}\left(x^{\alpha+1}\right)^{-2 \beta-1}\left[1-\left(x^{\alpha}+1\right)^{-2 \beta}\right]^{\theta-1} \text {; } \\
& f_{\text {KwBXIID }}(x)=\frac{a b \alpha \beta x^{\alpha-1}}{\left(x^{\alpha}+1\right)}\left[1-\left(x^{\alpha}+1\right)^{-\beta}\right]^{a-1}\left\{1-\left[1-\left(x^{\alpha}+1\right)^{-\beta}\right]^{a}\right\}^{b-1} ; \\
& f_{\text {BBXIID }}(x)=\alpha \beta[B(a, b)]^{-1} x^{\alpha-1}\left(x^{\alpha}+1\right)^{-\beta(b+1)}\left[1-\left(x^{\alpha}+1\right)^{-\beta}\right]^{a-1} \text {; } \\
& f_{\text {BEBXIDD }}(x)=\alpha \beta \theta[B(a, b)]^{-1} x^{\alpha-1}\left(x^{\alpha}+1\right)^{-\beta-1}\left[1-\left(x^{\alpha}+1\right)^{-\beta}\right]^{a \theta-1} \\
& \times\left\{1-\left[1-\left(x^{\alpha}+1\right)^{-\beta}\right]^{\theta}\right\}^{b-1} \\
& f_{\text {FBEBXIID }}(x)=\alpha \beta \theta^{-\alpha}[B(a, b)]^{-1} x^{\alpha-1}\left[1+\left(\frac{x}{\theta}\right)^{\alpha}\right]^{-\beta b-1}\left\{1-\left[1+\left(\frac{x}{\theta}\right)^{\alpha}\right]^{-\beta}\right\}^{a-1} \\
& f_{\text {FKwBXIID }}(x)=a b \alpha \beta x^{\alpha-1}\left[1+\left(\frac{x}{\theta}\right)^{\alpha}\right]^{-(\beta+1)}\left[1-\left(1+\left(\frac{x}{\theta}\right)^{\alpha}\right)^{-\beta}\right]^{a-1} \\
& \times\left\{1-\left[1-\left(1+\left(\frac{x}{\theta}\right)^{\alpha}\right)^{-\beta}\right]^{a}\right\}^{b-1} \\
& f_{\text {ZBBXIID }}(x)=\alpha \beta \Gamma^{-1}(a) x^{\alpha-1}\left(x^{\alpha}+1\right)^{-\beta-1}\left[1-\log \left(x^{\alpha}+1\right)^{-\beta}\right]^{a-1} \text {. }
\end{aligned}
$$

The parameters of the above densities are all positive real numbers and $x>0$. 


\section{Appendix B}

\section{Data Set I}

$\{0.98,5.56,5.08,0.39,1.57,3.19,4.90,2.93,2.85,2.77,2.76,1.73,2.48,3.68,1.08,3.22,3.75,3.22,3.70,2.74$, $2.73,2.50,3.60,3.11,3.27,2.87,1.47,3.11,4.42,2.40,3.15,2.67,3.31,2.81,2.56,2.17,4.91,1.59,1.18,2.48,2.03$, $1.69,2.43,3.39,3.56,2.83,3.68,2.00,3.51,0.85,1.61,3.28,2.95,2.81,3.15,1.92,1.84,1.22,2.17,1.61,2.12,3.09$, $2.97,4.20,2.35,1.41,1.59,1.12,1.69,2.79,1.89,1.87,3.39,3.33,2.55,3.68,3.19,1.71,1.25,4.70,2.88,2.96,2.55$, $2.59,2.97,1.57,2.17,4.38,2.03,2.82,2.53,3.31,2.38,1.36,0.81,1.17,1.84,1.80,2.05,3.65\}$.

\section{Data Set II}

$\{5.9,20.4,14.9,16.2,17.2,7.8,6.1,9.2,10.2,9.6,13.3,8.5,21.6,18.5,5.1,6.7,17,8.6,9.7,39.2,35.7,15.7,9.7,10$, $4.1,36,8.5,8,9.2,26.2,21.9,16.7,21.3,35.4,14.3,8.5,10.6,19.1,20.5,7.1,7.7,18.1,16.5,11.9,7,8.6,12.5,10.3$, $11.2,6.1,8.4,11,11.6,11.9,5.2,6.8,8.9,7.1,10.8\}$. 